\title{
COMPACT WELL-BOUNDED OPERATORS
}

\author{
CHENG QINGPING \\ School of Mathematical and Physical Sciences, Murdoch University, Murdoch, WA 6150, Australia \\ e-mail: cheng@prodigal.murdoch.edu.au \\ and IAN DOUST \\ School of Mathematics, University of New South Wales, Sydney, NSW 2052, Australia \\ e-mail: i.doust@unsw.edu.au
}

\begin{abstract}
Every compact well-bounded operator has a representation as a linear combination of disjoint projections reminiscent of the representation of compact self-adjoint operators. In this note we show that the converse of this result holds, thus characterizing compact well-bounded operators. We also apply this result to study compact well-bounded operators on some special classes of Banach spaces such as hereditarily indecomposable spaces and certain spaces constructed by G. Pisier.
\end{abstract}

1991 Mathematics Subject Classification. Primary 47B40. Secondary 34L10, 47A60, 47B07.

1. Introduction. Well-bounded operators are defined as those which possess a functional calculus for the absolutely continuous functions on some compact interval $[a, b]$ of the real line. Well-bounded operators were introduced by Smart [17] and Ringrose [14] in order to provide a theory for Banach space operators that was similar to the successful theory of self-adjoint operators on Hilbert space, but which included operators whose spectral expansions may only converge conditionally.

On a general Banach space the integral representation theorems which one obtains for these operators are much less satisfactory than those obtained for selfadjoint operators. Nonetheless, even on an arbitrary Banach space, every compact well-bounded operator can be written in the form

$$
T=\sum_{j=1}^{\infty} \lambda_{j} P_{j},
$$

where $\left\{\lambda_{j}\right\}$ is a sequence of real numbers converging monotonely in absolute value to 0 , and $\left\{P_{j}\right\}$ is a uniformly bounded sequence of disjoint projections. (See [3], [11, Theorem 4.2.3] or the closely related [2, Theorem 5.37].) This sum converges in the norm of $B(X)$, but possibly only conditionally. The aim of this paper is to provide a characterization of compact well-bounded operators via such representations. The

Some of this work forms part of the PhD research of the first author undertaken at Murdoch University under the supervision of Ken Harrison. The research of the second author was supported by the Australian Research Council. 
main point that we want to emphasize here is that under rather mild hypotheses on $\left\{\lambda_{j}\right\}$ and $\left\{P_{j}\right\}$, the sum appearing in the right-hand-side of $(*)$ always converges and that the limit is always a well-bounded operator. This has been an important element in several recent constructions. (See, for example, [4] and [6].)

In Sections 4 and 5, we use these result to examine compact well-bounded operators on some special classes of Banach spaces, such as hereditarily indecomposable spaces and certain spaces constructed by G. Pisier in [10].

2. Background and notation. In this section we shall give some of the basic definitions regarding well-bounded operators. The theory of well-bounded operators is given in more detail in [5].

Throughout $X$ will denote a complex Banach space with dual space $X^{*}$. The Banach algebra of all bounded linear operators on $X$ will be denoted by $B(X)$. The Banach algebra of absolutely continuous functions on the compact interval $[a, b] \subset \mathbb{R}$ will be denoted by $A C[a, b]$.

An operator $T \in B(X)$ is said to be well-bounded if there exist a constant $K$ and a compact interval $[a, b] \subset \mathbb{R}$ such that $\|p(T)\| \leq K\left\{|p(a)|+\int_{a}^{b}\left|p^{\prime}(t)\right| d t\right\}$, for all polynomials $p$. Equivalently, $T$ should possess a bounded functional calculus for $A C[a, b]$. That is, there should exist a Banach algebra homomorphism $f \mapsto f(T)$ (extending the natural definition for polynomials) such that

$$
\|f(T)\| \leq K\left\{|f(a)|+\operatorname{var}_{[a, b]} f\right\} \equiv K\|f\|_{A C}
$$

General well-bounded operators have an integral representation with respect to a family of projections on $X^{*}$ known as a 'decomposition of the identity'. This is a uniformly bounded, increasing family $\{F(\lambda)\}_{\lambda \in \mathbb{R}} \subset B\left(X^{*}\right)$ that satisfies certain rather complicated regularity conditions. We refer the reader to [15], [7] or [3] for full definitions.

Given a decomposition of the identity $\{F(\lambda)\}$, there exists a unique well-bounded operator $T \in B(X)$ such that

$$
\left\langle T x, x^{*}\right\rangle=b\left\langle x, x^{*}\right\rangle-\int_{a}^{b}\left\langle x, F(\lambda) x^{*}\right\rangle d \lambda \quad\left(x \in X, x^{*} \in X^{*}\right) .
$$

Every well-bounded operator has such a representation, but in general the decomposition of the identity is not uniquely determined by $T$. The well-bounded operator $T$ associated with a decomposition of the identity $\{F(\lambda)\}$ is of type $(A)$ if there is a family of projections $\{E(\lambda)\} \subset B(X)$ such that $F(\lambda)=E(\lambda)^{*}$ for all $\lambda \in \mathbb{R}$. (See $[\mathbf{1}$, Theorem 3.2]). Further, $T$ is said to be of type (B) if $E$ is right continuous in the strong operator topology and has a strong left hand limit at each point in $\mathbb{R}$. In this case, $\{E(\lambda)\}$ is called a spectral family concentrated on $[a, b]$.

3. Representations of compact well-bounded operators. The sums involved in the representation theorems are typically only conditionally convergent and so some care is needed in rearranging things. We shall need the following lemma about 
summation in topological vector spaces. Suppose that $\left\{x_{j}\right\}$ and $\left\{y_{j}\right\}$ are subsequences of a sequence $\left\{z_{j}\right\}$ with $x_{j}=z_{k(j)}$ and $y_{j}=z_{\ell(j)}$. Let $N_{x}=\{k(j): j \in \mathbb{N}\}$ and $N_{y}=\{\ell(j): j \in \mathbb{N}\}$. We shall say that $\left\{x_{j}\right\}$ and $\left\{y_{j}\right\}$ are complementary subsequences of $\left\{z_{j}\right\}$ if $N_{x}$ and $N_{y}$ are complementary subsets of $\mathbb{N}$. The relatively straightforward proof of Lemma 3.1 is left to the reader.

Lemma 3.1. Suppose that $\left\{z_{j}\right\}$ is a sequence in a topological vector space $Z$ and that $\left\{x_{j}\right\}$ and $\left\{y_{j}\right\}$ are complementary subsequences of $\left\{z_{j}\right\}$. If $\sum_{j=1}^{\infty} x_{j}$ and $\sum_{j=1}^{\infty} y_{j}$ both converge, then $\sum_{j=1}^{\infty} z_{j}$ converges and $\sum_{j=1}^{\infty} z_{j}=\sum_{j=1}^{\infty} x_{j}+\sum_{j=1}^{\infty} y_{j}$.

We shall now define hypotheses on a sequence of numbers $\left\{\lambda_{j}\right\}$ and a sequence of projections $\left\{P_{j}\right\}$ so that if $T \in B(X)$ can be written as $T=\sum_{j=1}^{\infty} \lambda_{j} P_{j}$ then $T$ is well-bounded.

(H1) $\left\{\lambda_{j}\right\}$ is a sequence of distinct real numbers such that $\left|\lambda_{1}\right| \geq\left|\lambda_{2}\right| \geq \cdots \rightarrow 0$.

(H2) $\left\{P_{j}\right\}$ is a sequence of disjoint finite-rank projections.

By disjoint we mean that $P_{i} P_{j}=0$ for $i \neq j$. By (H1) we can write $\left\{\lambda_{j}\right\}=\left\{\pi_{j}\right\} \cup\left\{v_{j}\right\}$ where $\pi_{1}>\pi_{2}>\ldots>0$ and $v_{1}<v_{2}<\ldots<0$. (Of course, one or more of these sets could be finite, or indeed empty. We shall leave it to the reader to make the necessary notational adjustments for these cases.) Let $P_{j}^{+}$be the projection corresponding to the eigenvalue $\pi_{j}$, and let $P_{j}^{-}$be the projection corresponding to $v_{j}$. For $n \geq 1$ let $Q_{n}^{+}=\sum_{j=1}^{n} P_{j}^{+}$and $Q_{n}^{-}=\sum_{j=1}^{n} P_{j}^{-}$. Our third hypothesis is that these operators are uniformly bounded.

(H3) There exists $K$ such that for all $n \geq 1,\left\|Q_{n}^{+}\right\| \leq K$ and $\left\|Q_{n}^{-}\right\| \leq K$.

Clearly (H3) implies that the projections $P_{j}$ are also uniformly bounded.

The main strength of the above hypotheses is that they ensure that the positive and negative parts can be summed separately. Once rearranged in this way, the construction of an $A C$ functional calculus is relatively straightforward.

Lemma 3.2. Suppose that $\left\{\lambda_{j}\right\}$ and $\left\{P_{j}\right\}$ satisfy (H1), (H2) and (H3) above. Then $\sum_{j=1}^{\infty} v_{j} P_{j}^{-}$and $\sum_{j=1}^{\infty} \pi_{j} P_{j}^{+}$both converge in norm.

Proof. Fix $m \leq n$. Then standard calculations show that

$$
\begin{aligned}
\left\|\sum_{j=m}^{n} v_{j} P_{j}^{-}\right\| & =\left\|v_{n} Q_{n}^{-}+\left(v_{n-1}-v_{n}\right) Q_{n-1}^{-}+\ldots+\left(v_{m}-v_{m+1}\right) Q_{1}^{-}\right\| \\
& \leq K\left(\left|v_{n}\right|+\left|v_{n-1}-v_{n}\right|+\ldots+\left|v_{m}-v_{m+1}\right|\right) \\
& =K\left|v_{n}-v_{m}\right| .
\end{aligned}
$$

It follows that the sequence $\sum_{j=1}^{n} v_{j} P_{j}^{-}$is Cauchy and hence it converges. The second sum is dealt with similarly.

Recall that a Riesz operator is one whose spectral theory mimics that of a compact operator. We refer the reader to [6, Chapter 3] for a more precise definition. 
Theorem 3.3 Suppose that $T \in B(X)$. Then the following are equivalent.

(i) $T$ is a compact well-bounded operator.

(ii) $T$ is a well-bounded Riesz operator.

(iii) $T=\sum_{j=1}^{\infty} \lambda_{j} P_{j}$, where $\left\{\lambda_{j}\right\}$ and $\left\{P_{j}\right\}$ satisfy $(\mathrm{H} 1)$, (H2) and (H3).

(iv) $T=\sum_{j=1}^{\infty} v_{j} P_{j}^{-}+\sum_{j=1}^{\infty} \pi_{j} P_{j}^{+}$where $\left\{v_{j}\right\}$ and $\left\{\pi_{j}\right\}$ are respectively sequences of negative and positive numbers satisfying $(\mathrm{H} 1)$, and $\left\{P_{j}^{-}\right\} \cup\left\{P_{j}^{+}\right\}$satisfies (H2) and (H3).

REMARK 3.4. We remind the reader that the sums appearing in Theorem 3.3 could be finite or (in condition (iv)) empty.

Proof. (i) $\Rightarrow$ (ii). This is trivial.

(ii) $\Rightarrow$ (iii). Suppose that $T$ is a well-bounded Riesz operator. That $T$ can be written as a sum of the required type and that $\left\{\lambda_{j}\right\}$ and $\left\{P_{j}\right\}$ satisfy (H1) and (H2) can be proved as in [3, Theorem 3.4]. It remains then to prove that the partial sums of the projections are uniformly bounded (i.e. (H3)). Let $g \in A C\left[v_{1}, \pi_{1}\right]$ be defined by

$$
g(\lambda)= \begin{cases}1 & \left(\lambda<v_{n}\right), \\ \frac{\lambda-v_{n+1}}{v_{n}-v_{n+1}} & \left(\lambda \in\left[v_{n}, v_{n+1}\right]\right), \\ 0 & \left(\lambda>v_{n+1}\right) .\end{cases}
$$

Note that if $\{F(\lambda)\}$ is any decomposition of the identity for $T$, then $F(\lambda)=\left(Q_{n}^{-}\right)^{*}$ for $\lambda \in\left[v_{n}, v_{n+1}\right)$. Thus, using Theorem 15.7 of [5], we have that for $x \in X$ and $x^{*} \in X^{*}$,

$$
\begin{aligned}
\left\langle g(T) x, x^{*}\right\rangle & =g\left(\pi_{1}\right)\left\langle x, x^{*}\right\rangle-\int_{v_{1}}^{\pi_{1}}\left\langle x, F(\lambda) x^{*}\right\rangle g^{\prime}(\lambda) d \lambda \\
& =-\int_{v_{n}}^{v_{n+1}}\left\langle Q_{n}^{-} x, x^{*}\right\rangle\left(\frac{1}{v_{n}-v_{n+1}}\right) d \lambda \\
& =\left\langle Q_{n}^{-} x, x^{*}\right\rangle .
\end{aligned}
$$

Thus $\left\|Q_{n}^{-}\right\| \leq K\|g\|_{B V}=K$. A similar proof shows that the projections $Q_{n}^{+}$are also uniformly bounded.

(iii) $\Rightarrow$ (iv). This follows from Lemmas 3.1 and 3.2.

(iv) $\Rightarrow$ (i). Since $T$ is the sum of norm limits of finite rank operators, it is clearly compact. It remains then to show that $T$ admits an $A C\left[v_{1}, \pi_{1}\right]$ functional calculus. Suppose that $g \in A C\left[v_{1}, \pi_{1}\right]$. Then $g=g^{-}+g_{0}+g^{+}$, where $g^{-}=0$ on $\left[0, \pi_{1}\right], g_{0}$ is constant and $g^{+}=0$ on $\left[v_{1}, 0\right]$. We claim that, for all $g \in A C\left[v_{1}, \pi_{1}\right]$,

$$
S_{1}=\sum_{j=1}^{\infty} g^{-}\left(v_{j}\right) P_{j}^{-} \quad \text { and } \quad S_{2}=\sum_{j=1}^{\infty} g^{+}\left(\pi_{j}\right) P_{j}^{+}
$$

converge in norm and that setting

$$
g(T)=\sum_{j=1}^{\infty} g^{-}\left(v_{j}\right) P_{j}^{-}+g_{0}(0) I+\sum_{j=1}^{\infty} g^{+}\left(\pi_{j}\right) P_{j}^{+}
$$


defines an algebra homomorphism from $A C\left[v_{1}, \pi_{1}\right]$ to $B(X)$. Note that by rearranging the sum we get

$$
\sum_{j=1}^{n} g^{-}\left(v_{j}\right) P_{j}^{-}=g^{-}\left(v_{n}\right) Q_{n}^{-}+\sum_{j=1}^{n-1}\left(g^{-}\left(v_{j}\right)-g^{-}\left(v_{j+1}\right)\right) Q_{j}^{-} .
$$

Since $g^{-}\left(v_{n}\right) \rightarrow 0$ as $n \rightarrow \infty$ and $\sum_{j=1}^{n-1}\left|g^{-}\left(v_{j}\right)-g^{-}\left(v_{j+1}\right)\right| \leq \operatorname{var}_{\left[v_{1}, 0\right]} g$, the series $S_{1}$ converges. The other series, $S_{2}$, is dealt with similarly.

The map $g \mapsto g(T)$ is clearly linear and, since

$$
\|g(T)\| \leq K \underset{\left[\nu_{1}, 0\right]}{\operatorname{var}} g+|g(0)|+K \underset{\left[0, \pi_{1}\right]}{\operatorname{var}} g \leq K^{\prime}\|g\|_{A C},
$$

it is also bounded. One readily verifies that if $p$ is a polynomial, then the definition of $p(T)$ given by (1) agrees with the natural polynomial functional calculus. It follows that the map $g \mapsto g(T)$ is an algebra homomorphism, and hence that $T$ has an $A C\left[v_{1}, \pi_{1}\right]$ functional calculus.

Theorem 3.3 can clearly be used to construct well-bounded operators. Indeed this result is the basis for constructions in [4] and [6].

Corollary 3.5. Suppose that $\left\{\lambda_{j}\right\}$ and $\left\{P_{j}\right\}$ satisfy hypotheses $(\mathrm{H} 1)$, (H2) and (H3). Then the series $\sum_{j=1}^{\infty} \lambda_{j} P_{j}$ converges in norm, and the sum is a compact wellbounded operator.

Proof. By Lemma 3.2, $\sum_{j=1}^{\infty} v_{j} P_{j}^{-}$and $\sum_{j=1}^{\infty} \pi_{j} P_{j}^{+}$converge. Let $T=\sum_{j=1}^{\infty} v_{j} P_{j}^{-}+$ $\sum_{j=1}^{\infty} \pi_{j} P_{j}^{+}$. By Theorem 3.3, $T=\sum_{j=1}^{\infty} \lambda_{j} P_{j}$ and $T$ is compact and well-bounded.

REMARK 3.6. The situation for operators with complex spectrum is rather less clear. In [8] it was shown that compact operators with an $A C$ functional calculus for a rectangle in the plane (i.e. compact $A C$-operators) admit a representation in the form $T=\sum \lambda_{j} P_{j}$. We do not know if there is any reasonable characterization of compact $A C$-operators in terms of such representations.

Proposition 3.7. Suppose that $T \in B(X)$ is a compact well-bounded operator such that $T=\sum_{j=1}^{\infty} \lambda_{j} P_{j}$, where $\left\{\lambda_{j}\right\}=\left\{v_{j}\right\} \cup\left\{\pi_{j}\right\},\left\{P_{j}\right\},\left\{Q_{j}^{-}\right\}$and $\left\{Q_{j}^{+}\right\}$satisfy $(\mathrm{H} 1),(\mathrm{H} 2)$ and (H3). Then $T$ is of type ( $A)$ if and only if $\lim _{n \rightarrow \infty} Q_{n}^{+}$exists in the strong operator topology of $B(X)$. Further, $T$ is of type (B) if and only if both $\lim _{n \rightarrow \infty} Q_{n}^{-}$and $\lim _{n \rightarrow \infty} Q_{n}^{+}$exist in the strong operator topology.

Proof. Suppose that $T$ is of type (A). One can check that if $\{E(\lambda)\}$ is any family of projections such that $\left\{E(\lambda)^{*}\right\}$ forms a decomposition of the identity for $T$, then $E(\lambda)=Q_{n}^{-}$for $\lambda \in\left[v_{n}, v_{n+1}\right)$ and $E(\lambda)=Q_{n}^{+}$for $\lambda \in\left[\pi_{n+1}, \pi_{n}\right)$. Thus $\lim _{\lambda \rightarrow 0^{+}} E(\lambda)=\lim _{n \rightarrow \infty} Q_{n}^{+}$exists in the strong operator topology. Conversely, if $\lim _{n \rightarrow \infty} Q_{n}^{+}$exists, define $E(0)=\lim _{n \rightarrow \infty} Q_{n}^{+}, E(\lambda)=Q_{n}^{-}$for $\lambda \in\left[v_{n}, v_{n+1}\right)$ and $E(\lambda)=Q_{n}^{+}$for $\lambda \in\left[\pi_{n+1}, \pi_{n}\right)$. Then $\left\{E(\lambda)^{*}\right\}$ is a decomposition of the identity for $T$ and so $T$ is of type (A), by Theorem 3.2 of [1].

Similarly, $T$ has a spectral family if and only if both $\lim _{n \rightarrow \infty} Q_{n}^{-}$and $\lim _{n \rightarrow \infty} Q_{n}^{+}$ exist. 
4. Well-bounded operators and Schauder decompositions. Let $X$ be a Banach space. A sequence of projections $\left\{P_{n}\right\}_{n=1}^{\infty}$ in $B(X)$ is said to be a Schauder decomposition for $X$ if

(1) $P_{n} P_{m}=P_{\min (m, n)}$ for all integers $m, n \geq 1$;

(2) $P_{n} \rightarrow I$ in the strong operator topology;

(3) $P_{n} \neq P_{m}$ if $m \neq n$.

A Schauder decomposition $\left\{P_{n}\right\}_{n=1}^{\infty}$ for $X$ is said to be finite dimensional, if each $P_{n}$ is a finite-rank projection, for every $n \in \mathbb{N}$.

We shall say that a Banach space is a GDP-space if it is a Grothendieck space with the Dunford-Pettis property. Examples of GDP-spaces are $L^{\infty}$ and $H^{\infty}(\mathbb{D})$. It is well known that a GDP-space has no subspaces with a Schauder decomposition. (See, for example, Remark 15.3.(a) of [16].) Ricker [12] showed, using this fact, that on a GDP-space, every well-bounded operator of type (B) has a finite spectrum and hence is a scalar-type operator with real spectrum. The following theorem characterizes the Banach spaces on which every well-bounded operator of type (B) admits this property as exactly those spaces which do not contain any complemented subspace with Schauder decomposition.

Theorem 4.1. Suppose that $X$ is a Banach space. Then the following statements are equivalent:

(i) X contains no complemented subspace with a Schauder decomposition;

(ii) every well-bounded operator of type (B) on X has a finite spectrum.

Proof. A close examination of the proof of [12, Lemma 12] shows that (i) implies (ii).

Suppose now that (i) is false, i.e. that there exists a complemented subspace $Y$ of $X$ with Schauder decomposition $\left\{P_{n}\right\}_{n=1}^{\infty}$. Suppose also that $\left\{\lambda_{n}\right\}_{n=1}^{\infty}$ is a strictly increasing infinite sequence of real numbers converging to 0 . By Theorem 3.1 and Proposition 4.1 of [3], setting

$$
E(\lambda)= \begin{cases}0 & \left(\lambda<\lambda_{1}\right) \\ P_{n} & \left(\lambda \in\left[\lambda_{n}, \lambda_{n+1}\right]\right), \\ I & (\lambda \geq 0)\end{cases}
$$

defines the spectral family of a well-bounded operator of type (B), say $T_{0} \in B(Y)$. It is easy to see that $\sigma\left(T_{0}\right)=\left\{\lambda_{n}\right\}_{n=1}^{\infty} \cup\{0\}$. Now define an operator $T$ on $X$ by $T=T_{0} \oplus 0$. It follows from Lemma 4.3 of [3] that $T$ is also well-bounded of type (B) and clearly $T$ has infinite spectrum. It follows that (ii) implies (i).

The following theorem should be compared to Theorem 4.1.

Theorem 4.2. Suppose that $X$ is a Banach space. Then the following statements are equivalent:

(i) X contains no complemented subspace with a finite dimensional Schauder decomposition;

(ii) every compact well-bounded operator of type (B) on $X$ has a finite spectrum. 
Proof. Suppose that (i) holds. Let $T \in B(X)$ be a compact well-bounded operator of type (B). By Theorem 3.3,

$$
T=\sum_{j=1}^{\infty} v_{j} P_{j}^{-}+\sum_{j=1}^{\infty} \pi_{j} P_{j}^{+},
$$

where $\left\{v_{j}\right\}$ and $\left\{\pi_{j}\right\}$ are respectively sequences of negative and positive numbers satisfying (H1), and $\left\{P_{j}^{-}\right\} \cup\left\{P_{j}^{+}\right\}$satisfies (H2) and (H3). Thus $\sigma(T)=\left\{v_{j}\right\} \cup\left\{\pi_{j}\right\} \cup\{0\}$. We shall show that neither $\left\{v_{j}\right\}$ nor $\left\{\pi_{j}\right\}$ can be infinite sets. Suppose that $\left\{v_{j}\right\}$ is an infinite set. For $n \geq 1$ let $Q_{n}^{-}=\sum_{j=1}^{n} P_{j}^{-}$. Since $T$ is of type (B), it is easy to check that if $\{E(\lambda)\}$ is a family of projections on $X$ whose adjoints form a decomposition of the identity for $T$, then $E(\lambda)=Q_{n}^{-}$for $\lambda \in\left[v_{n}, v_{n+1}\right)$. Thus the limit

$$
E(0-)=\lim _{\lambda \rightarrow 0^{-}} E(\lambda)=\lim _{n \rightarrow \infty} Q_{n}^{-}
$$

exists in the strong operator topology, and $Y=E(0-) X$ is a complemented subspace of $X$. It is easy to check that $\left\{Q_{n}^{-} \mid Y\right\}_{n=1}^{\infty}$ forms a finite dimensional Schauder decomposition for $Y$, which is infinite dimensional, since $\left\{v_{j}\right\}$ is an infinite set. This is contrary to (i). Hence $\left\{v_{j}\right\}$ is a finite subset. It can be argued similarly that $\left\{\pi_{j}\right\}$ is also a finite subset. Thus (i) implies (ii).

Suppose now that $X$ contains a complemented subspace, say $Y$, that admits a finite dimensional Schauder decomposition $\left\{Q_{n}\right\}_{n=1}^{\infty}$. Let $P_{1}=Q_{1}, P_{n}=Q_{n}-Q_{n-1}$ for $n \geq 2$ and let $\left\{v_{n}\right\}_{n=1}^{\infty}$ be a strictly increasing sequence of real numbers converging to 0 . It is obvious that $\left\{P_{n}\right\}_{n=1}^{\infty}$ and $\left\{v_{n}\right\}_{n=1}^{\infty}$ satisfy (H1), (H2) and (H3). By Corollary 3.5, the series $\sum_{n=1}^{\infty} v_{n} P_{n}$ converges in norm, and the sum $T_{0}$ is a compact well-bounded operator on $Y$. By Proposition 3.7, $T_{0}$ is of type (B). As before $T=T_{0} \oplus 0$ is a compact well-bounded operator of type (B) on $X$ with infinite spectrum. This shows that (ii) implies (i).

REMARK 4.3. We do not know whether Condition (ii) in Theorem 4.2 can be strengthened in some way. It cannot be replaced by the condition that every compact well-bounded operator of type (A) on $X$ has a finite spectrum.

To see this, note that a Banach space $X$ with a finite dimensional Schauder decomposition is separable, and that no separable subspace of $\ell^{\infty}$ is complemented in $\ell^{\infty}$. Hence $\ell^{\infty}$ satisfies (i) of Theorem 4.2. Define a well-bounded operator $T$ on $\ell^{\infty}$ by $T\left(x_{1}, x_{2}, \ldots\right)=\left(\frac{1}{2} x_{1}, \frac{2}{3} x_{2}, \ldots, \frac{n-1}{n} x_{n}, \ldots\right)$. Then $T$ is a compact well-bounded operator of type (A) with infinite spectrum, by [1, Proposition 6.5].

5. Well-bounded operators on some special spaces. In [10] Pisier gave a construction which showed that there exists a Banach space $X$ with the following properties:

(1) $X$ is nonreflexive;

(2) there exists a positive constant $c$ such that if $P$ is a projection onto a subspace of dimension $n$, then $\|P\| \geq c n^{\frac{1}{2}}$.

We shall say that a Banach space $X$ is a Pisier space if $X$ has these two properties. 
A longstanding open question in the theory of well-bounded operators is whether there are any nonreflexive Banach spaces on which every well-bounded operator is of type (B). It was shown in [3] that if $X$ is a Banach space which admits a complemented nonreflexive subspace with a basis, then there exists a well-bounded operator on $X$ which is not of type (B). Indeed the proof shows that on such a space one can always find a compact well-bounded operator which is not of type (B). Obviously, this theorem does not tell us what happens on Pisier spaces. The following theorem shows that one will need to use quite different methods if one is to construct a well-bounded operator on a Pisier space that is not of type (B).

Theorem 5.1. Suppose that $X$ is a Pisier space. Every compact well-bounded operator $T \in B(X)$ has a finite spectrum and hence is of type $(B)$.

Proof. Let $T$ be a compact well-bounded operator on $X$. By Theorem 3.3, $T$ has the form

$$
T=\sum_{j=1}^{\infty} v_{j} P_{j}^{-}+\sum_{j=1}^{\infty} \pi_{j} P_{j}^{+},
$$

where $\left\{v_{j}\right\}$ and $\left\{\pi_{j}\right\}$ are respectively sequences of negative and positive numbers satisfying (H1), and $\left\{P_{j}^{-}\right\} \cup\left\{P_{j}^{+}\right\}$satisfies (H2) and (H3). By (H3) and condition (2) for Pisier space, both $\left\{P_{j}^{-}\right\}$and $\left\{P_{j}^{+}\right\}$are finite sets. Hence $T$ has a finite spectrum and so $T$ is of type (B).

We shall now discuss well-bounded operators in the class of hereditarily indecomposable Banach spaces. Recall that a Banach space $X$ is said to be hereditarily indecomposable (briefly, H.I.) if it has the property that, whenever $Y$ and $Z$ are closed, infinite dimensional subspaces of $X$ and $\delta>0$, then there exist unit vectors $f \in Y$ and $g \in Z$ such that $\|f-g\|<\delta$. This is equivalent to the condition that no subspace of $X$ can be written as a topological direct sum of two closed, infinite dimensional subspaces.

In 1993, Gowers and Maurey [9] gave the first example of a H.I. Banach space in their solution to the unconditional basis sequence problem. This space is not only reflexive, but it has a basis. Since then a number of H.I. spaces have been constructed (using methods similar to the one of Gowers and Maurey in [9]) for a variety of purposes. The space of operators on a H.I. space $X$ is very small: every bounded linear operator on $X$ can be written as $\lambda I+S$, where $S$ is a strictly singular operator. It is possible that Gowers and Maurey's space provides an example of a Banach space on which every bounded linear operator is of the form $\lambda I+K$ for a compact operator $K$, although this question was left open in [9].

Ricker [13, Theorems 1-5] gave a complete description of the structure and spectral properties of well-bounded operators of type (B) on H.I. spaces. In particular, it was proved that every well-bounded operator $T$ of type (B) on such a space can be written as $T=\lambda I+K$, where $\lambda$ is a scalar and $K$ is a compact operator. In fact, this result holds not only for well-bounded operators of type (B), but also for general well-bounded operators.

Theorem 5.2. Suppose that $X$ is a H.I. space and $T$ is a well-bounded operator on $X$. Then there exists a compact operator $K \in B(X)$ and a constant $\lambda \in \mathbb{R}$ such that $T=\lambda I+K$. Furthermore, this representation is unique. 
Proof. By Lemma 2 (ii) of [13], there exists a unique point $\lambda \in \sigma_{e}(T)$ and if $K=T-\lambda I$, then $K$ is a Riesz operator. But if $T$ is well-bounded, then so is $K$. By Theorem 3.3, $K$ must be compact. The uniqueness is clear.

\section{REFERENCES}

1. B. Ashton, Cheng Qingping and I. Doust, Some remarks on well-bounded and scalar-type decomposable operators, Houston J. Math., to appear.

2. H. Benzinger, E. Berkson and T. A. Gillespie, Spectral families of projections, semigroups, and differential operators, Trans. Amer. Math. Soc. 275 (1983), 431-475.

3. Cheng Qingping and I. Doust, Well-bounded operators on nonreflexive Banach spaces, Proc. Amer. Math. Soc. 126 (1996), 799-808.

4. Cheng Qingping and I. Doust, The dual theory of well-bounded operators, $J$. Operator Theory 37 (1997), 35-50.

5. H. R. Dowson, Spectral theory of linear operators, London Mathematical Society Monographs No. 12 (Academic Press, London, 1978).

6. I. Doust and T. A. Gillespie, An example in the theory of $A C$-operators, Proc. Amer. Math. Soc. 129 (2001), 1453-1457.

7. I. Doust and Qiu Bozhou, The spectral theorem for well-bounded operators, J. Austral. Math. Soc. (Series A), 54 (1993), 334-351.

8. I. Doust and B. L. Walden, Compact AC-operators, Studia Math. 117 (1996), 275287.

9. W. T. Gowers and B. Maurey, The unconditional basic sequence problem, J. Amer. Math. Soc. 6 (1993), 851-874. $181-208$

10. G. Pisier, Counterexamples to a conjecture of Grothendieck, Acta Math. 151 (1983),

11. D. J. Ralph, Semigroups of well-bounded operators and multipliers, Thesis, University of Edinburgh, 1977.

12. W. Ricker, Well-bounded operators of type (B) in a class of Banach spaces, J. Austral. Math. Soc. (Series A) 42 (1987), 399-408.

13. W. Ricker, Well-bounded operators of type (B) in H.I. spaces, Acta Sci. Math. (Szeged) 59 (1994), 475-488.

14. J. R. Ringrose, On well-bounded operators, J. Austral. Math. Soc. (Series A) 1 (1960), 334-343.

15. J. R. Ringrose, On well-bounded operators II, Proc. London Math. Soc. (3) 13 (1963), 613-638.

16. I. Singer, Bases in Banach spaces (Springer-Verlag, 1970).

17. D. R. Smart, Conditionally convergent spectral expansions, J. Austral. Math. Soc. (Series A) 1 (1960), 319-333. 